\title{
A Study on the Problems of Mac Protocol in WSN'S
}

\author{
Aiman J. Albarakati, \\ Department of Computer Engineering, \\ College of Computer and Information Science, \\ Majmaah University, Majmaah, Saudi Arabia
}

\begin{abstract}
In recent years there has been a growing interest in Wireless Sensor Networks (WSN). Recent advancements in the field of sensing, computing and communications have attracted research efforts and huge investments from various quarters in the field of WSN. Also sensing networks will reveal previously unobserved phenomena. The various areas where major research activities going on in the field of WSN are deployment, localization, synchronization, data aggregation, dissemination, database querying, architecture, middleware, security, designing less power consuming devices, abstractions and higher level algorithms for sensor specific issues. In past couple of year Medium Access Control (MAC) for WSN has been an incredibly vigorous research field. An alphabet broth of MAC protocol has been endowed by sensor networks Literature/prose with nearly all of the mechanism concerning merely on energy effectiveness. A great deal of additional ground-breaking work need to be prepared at the Medium Access Control layer, however contemporary exertions are not in positions of mapping the stiff unanswered tribulations. This paper is all about an overview of Medium Access Control Protocol concerns and prospects defies.
\end{abstract}

\section{Keywords}

WSN, Protocol, MAC

\section{INTRODUCTION}

If we have a close look over sensor networks so in past few years these have came forward as one of the prevailing technology drift. Wireless communications, embedded computing Sensors and actuators are not innovative perceptions or ideas however these are the up to date low-cost large-scale addition and incorporation of communication, computation and sensing into WSN's that has seized the concentration and interest of loads of researchers and scientists. Sensor Networks facilitate to keep an eye on the physical world at a granularity intensity which we would say was never came into being earlier than now [2]. Applications of sensor networks take in an assortment of order s and areas, restricted barely by the thoughts of the application developer. It is crucial during thinking process of future research prospects for wireless networks to mull over that which sort of devices would be associated to the concern network. Mainstream of the exertions coming into view in the literature or exploration are slightest publishable incremental enhancement over the well-liked S-MAC protocol [1]. In near term nearly all regular form of devices on the network would be entrenched processors, e.g. sensors and actuators and augmenting battery lifetime shall be extremely significant than boosting up performance [3]. As per Moore's law energy utilization by computing chips is declining stridently for every unit computation but energy put away by radios is settling on by physics laws. As a result, the wireless interface or boundary will be the main end user of energy in any device that merges radios and computations [3]. The MAC layer assembles unswervingly on apex of the Physical layer and be in command of the radio. It meeting point is energy competence in its place of meeting long-established objectives for wireless MAC plan like bandwidth utilization, fairness and delay [4]. These protocols tradeoffs recital for energy outlay. Major supplies of energy depletion at the Medium Access Control layer are impacts, idle listening, overhearing, and control packet overhead [4]. Nothing like the 802.11 WLAN cards where the Medium Access Control is by and large incorporated as part of the chipset in sensor networks the Medium Access Control designer has unconditional power on the Medium Access Control layer design. This supreme command with the reality that the wireless interface is the most important end user of battery in energy inhibited sensor networks, has prepared medium access for sensor networks an extremely vigorous research field. Nevertheless, up to date exploration on Medium Access Control protocols for sensornets or sensor networks scrutinize that there is no comprehensible inclination demonstrating that medium access for sensor-networks is coming together in the direction of a matchless preeminent elucidation [5]. There is a propensity of "re-inventing the wheel" in the Medium Access Control for sensor-networks field with mainstream of the efforts asserts a hardly any percent superior show over the fashionable SMAC [1] protocol. In its place of spotlight merely on energy effectiveness, it's era to deal with additional unsettled dilemmas at the Medium Access Control layer.

\section{RESEARCH PROBLEMS}

\subsection{Structural Design of Network}

The cross-layer devises or plans in sensor-networks have show the way to monumental, perpendicularly incorporated elucidations which may perhaps effort autonomously other than are not in actual fact functional for extra research groups. One of the premature heartening strides in the direction of sensor network structural design is sensornet protocol (SP) [6] which is a supple substitute to the up to date ZigBee design. sensornet protocol endow with a consistent interface to Medium Access Control with a little response in equally directions and affords a vital stride to edifice a better sensor network structural design. Contrasting IP in the Internet, sensornet protocol is not at the network layer nevertheless as a substitute sits between the data-link and network layer. Crafting sensor network architecture/structural design would be a mounting and unrefined course. Prospect Medium Access Control designers/developers ought to carry on the broadspectrum aspiration of approaching in the direction a sensor network structural design in psyche and endeavor to bring into play of the services that sensornet protocol be obliged to put forward [6].

\subsection{Uniform and Consistent Radio Hardware}

As we discussed above that [6] is the up-and-coming paradigm on top of the Medium Access Control layer in the sensor-net network stack, IEEE 802.15.4 is the promising 
archetype for inferior layers the physical and medium access. IEEE 802.15.4 archetype takes in both physical (PHY) and medium access control (MAC) provisions. Nevertheless, we look forward to that investigators would fundamentally supersede the 802.15.4 Medium Access Control and merely the 802.15.4 PHY standard would have insinuations on prospect sensor-net Medium Access Control plan. Innovative and forthcoming sensor-net hardware platforms, like Sun SPOTS platform "Sun Labs", Telos Motes "Moteiv" and MicaZ Motes "CrossBow", by now bring into play IEEE 802.15.4 acquiescent radios. By utilizing a consistent radio, the nodes are able to be in touch with any number of devices though sharing the identical physical layer. In contrast as the IEEE 802.15.4 radio interfaces are packet-based, the developers/designers mislay substantial suppleness in software for managing the radios.

\subsection{Passive or Reflexive Co-Existence}

By way of introduction o the projected large-scale sensornets into our everyday lives, a state of affairs will come into view where sensornets from unusual vendors/operators require maneuvering at an ordinary frequency band in the identical physical environment. This place an obligation on the Medium Access Control protocols to act pleasant to every protocol. Cleanly in succession at a squat duty-cycle is not sufficient to guarantee coexistence. Foremost, these protocols have to be aware of other protocols to promise unblemished function. far-off messages call for to be filtered out, obstruction recognition wants to be improved to stay away from offensive back offs, schedules call for to be attuned to shun overlie vigorous parts. Subsequent these are obliged to think about protection/security concerns to shield against snooping and nasty activities. Even though it will be slam to impracticable to put off denial-of-service assaults, perhaps as atrocious as overcrowding, scrutinize this and signaling it to the routing layer may possibly put off an entire malfunction of the application. [7] is an earliest stride in sheltering these protocols, however its dependence on a shared key constructs it defenseless and for that reason further sophisticated methods are desirable. Breakdown of nodes and network expansions are obscuring aspects that have acknowledged diminutive consideration in the protection field, nevertheless are indispensable to the flourishing maneuver of sensor-nets in the prospect. The hardware elucidations, similar to endowing manifold channels at the radio, may possibly assuage a number of the dilemmas of coexistence; however the safety measures concerns will at a standstill have to be determined.

\subsection{Mobile Sensor Networks}

The aspect of mobility at the Medium Access Control Layer has been generally ignored by the research society for the reason that sensor-nets were initially understood to encompass of stationary nodes. Nevertheless, latest explorations resembling [8] [9] have facilitated mobility in sensor-nets. In addition, in recent times there has been an amplified significance in therapeutic health or heed and catastrophe rejoinder applications of sensor networks and these atmospheres or settings brings into play mobile sensor nodes [10]. The merely explorations we can mull over, which unambiguously reflect on mobility at the Medium Access Control layer is [11] and we have much more interest of research in this particular field. [10] Mobility appraisals ought to reflect on further pragmatic mobility sculpts only regard as the emblematic faction of a doctor in hospice lobby, and [11] utilizes a comparatively straightforward indiscriminate mobility sculpt for simulations. In addition, all-inclusive authentic mobility marks out could bear out constructive in such mobility assessments.

\subsection{New-fangled Optimization decisive factor}

The initial devise purpose for sensor networks and Medium Access Control has been remained energy proficient till date. On the other hand, due to emergence of novel applications for sensor networks, other optimization conditions/parameters likewise latency and acquiescence with synchronized constrictions or steadfast data liberation e.g. medical applications may possibly put on consequence. A diminutive consideration has been compensated to them in the background of sensor networks. One finicky problem is that loads of applications call for optimization for manifold, incompatible conditions energy competent and steadfastness. For this reason, there must be a way for the applications to put into service fastidious trade-offs between these inconsistent aspirations. At this point, MAC protocols would be mandatory that endow with "turning knobs" to afford application-specific trade-offs.

\subsection{The Perception and Utilization of Traffic Patterns}

Majority capricious and random communication patterns have been supported by nearly all on hand Medium Access Control protocols for sensor networks are to a certain extent wideranging rationale in that they hold up. At the same time as this consent to the realization of subjective disseminated protocols, the suppleness outlay is frequently restricted competence. Nonetheless, loads of sensor networks applications demonstrate a little somewhat explicit traffic patterns such as put on air from the sink to all other sensor nodes and congregate shed from all nodes in the direction of the sink. As well as detailed patterns in sensor networks has been showed by Also, traffic generation. Whereas sensor networks for unremitting monitor display data streams from nodes to the sink, event-based applications engender traffic barely leading the incidence of confident motivating events. In view of the fact that real-world events are frequently concomitantly scrutinized by several nodes in the environs of the incident and likewise event-based applications over and over again upshot in decidedly allied invention of traffic in the network. Furthermore, confident traffic patterns time and again come into sight in phases like intermittent reservation distributions trail by extensive phases of data gathering or a rupture of event hearsay tag on by elongated inoperative phases. A great deal of efficiency of MAC protocols could possibly be achieved by improved indulgent and utilization such traffic patterns in the Medium Access Control plan.

\subsection{Painstaking detrimental of Simulation}

Postulations that have been crafted in good number simulation settings are referred as circular; all radios have identical range don't unavoidably be a sign of the real-world state of affairs. In view of that fact to entirely comprehend the intricacy of scheming a Medium Access Control protocol and to build up elucidations which effort in actual life then it is indispensable to not merely mock-up or imitate but in addition to put into practice and trial on real world systems as well. It is imperative to re-examine Kotz's erroneous proverbs of wireless network research [12] to be aware of why MAC protocols that acquiesce enormously perfect outcomes in simulation fall short in actual life exploitations [13]. Flat earth 
sculpts take for granted that two nodes may possibly flawlessly be in touch if they are inside a number of distance. [14] Assessed and illustrated that MANET simulation outcomes be deficient in truthfulness. Sensor-net researchers and scientists have to demeanor researches comparable to [12] and [14] on the sensor-net literature and make out the erroneous axiom/proverbs in the radio models utilized by trendy sensor-net simulation surroundings/settings.

As far as simulation is concern so in contrast to OMNeT++ simulator for sensor networks MAC simulations it is far superior to bring into play MAC Simulator 0.2.2 [15] that has pre-default code for comparison protocols in its library. TOSSIM [16] facilitates the Medium Access Control developers to pick the precision and intricacy of the radio sculpts as obligatory for their simulations. Nevertheless, like ns-2 (Network Simulator-2) and MAC Simulator 0.2.2, the on hand radio models in TOSSIM are comparatively straightforward. Emstar [17] lends a hand the sensor-net developers/Programmers to straightforwardly stir from simulation to archetype to employment.

\subsection{Revolution OF Software Radios:}

Software defined radios (SDR) may possibly be as innovative and alterative technology for wireless communication. The facets of SDR such as MAC, data indoctrination, frequency handling and so on are programmable in its place of hardwired and initiating new-fangled media access regulations turns out to be a subject of a trouble-free software alteration. As we know that the performance or recital outcomes of Medium Access Control protocols in simulations ought to be taken with a granule of saline for the reason that of probable impracticable postulations prepared in radio models of the simulation atmosphere. A probable way out more or less this dilemma could be to set up scheming for SDR systems and progress from simulation to prototypes. It also consent the network researchers/scientists to bring into play economical off-the-shelf networking cards to conduct test on novel MAC protocols. [18] Have proposed a software structural design SoftMAC, on top of the radio subsystem (IEEE 802.11 family network cards) that authorize network researchers/scientists to effortlessly put up and set up tentative self-motivated MAC layers.

\subsection{Experimental Realism}

Despite the fact that SDR are one imperative track headed for getting hold of more reasonable approaching into MAC layer recital/act, an additional stride is experimental realism or in other words we can refer it real-world experiments. Durable experiments like [19] are the majority pragmatic ones other than entails a lot of engineering exertion prior to study queries can be responded [13]. Proscribed covered trials or experiments are not as easy-to-read as across-the-board exploitations nevertheless in view of the fact that authentic radio hardware is utilized, they don't undergo from the erroneous axioms. [20] have anticipated to put back the batteries with towering faculty capacitors so-called GoldCaps for the reason of investigational justification of lifetime limits for WSN. Likewise it can also be bring into play to measure up to the energy effectiveness of Medium Access Control protocols devoid of coming up for the batteries of the sensor nodes to deplete. At the same time as this practice have intrinsic hitches, so for the same reason it is not able to acquire the battery leisure cause into relation and outcomes are much practical than simulations by utilizing this practice.

\section{CONCLUSION}

While appraising the unfasten concerns in the Medium Access Control protocols for sensor networks, it has been unraveled that there is no such apparent path or step through which prospect endeavors ought to be intended or headed. However a number of suggestions should be taken under consideration, Like, 1) Energy competence should not be the merely design ambition rater than taking other aspects under consideration as well. 2) By superior understanding and comprehension specific traffic patters can be utilized for arbitrary patters as well. 3) Security should be the top concern for protection against malevolent actions. 4) MAC protocols should be flexible, supple and adaptable to co-exist with other different vendors protocols on same frequency band. 5) Poor performance of MAC protocols for sensor networks with mobile sensor nodes should be addressed and must be resolve to overcome the performance issue. 6) There is a much more need of research and study in regards of simulation and standardization, in view of the fact that much more reasonable insight into MAC layer performance/recital or act is needed.

\section{REFERENCES}

[1] "W. Ye, J. Heidemann", “An energy-efficient mac protocol for wireless sensor networks," IEEE Infocom. New York, NY, USA: IEEE, June 2002.

[2] "J. Elson", Wireless Sensor Networks. Kluwer Academic Publishers, 2004, ch. Wireless Sensor Networks: A bridge to the Physical World.

[3] "D. Clark, C. Partridge, R. Braden, B. Davie", "Making the world of communications a difference place," ACM SIGCOMM Computer Communication Review, July 2005.

[4] "W. Ye and J. Heidemann", Wireless Sensor Networks. Kluwer Academic Publishers, 2004, ch. Medium Access Control in Wireless Sensor Networks/

[5] "K. Langendoen and G. Halkes," Embedded Systems Handbook. CRC Press, Aug. 2005, ch. Energy-Efficient Medium Access Control.

[6] "J. Polastre, J. Hui," "A unifying link abstraction for wireless sensor networks," ACM SenSys'05, Nov. 2005.

[7] "C. Karlof, N. Sastry", "TinySec: a link layer security architecture for wireless sensor networks," in Proc. SenSys'04, Nov. 2004.

[8] 'K. Dantu, M. Rahimi”, "Robomote: Enabling mobility in sensor networks," in IEEE/ACM Fourth International Conference on Information Processing in Sensor Networks (IPSN/SPOTS), April. 2005.

[9] "M. Laibowitz and J. A. Paradiso", "Parasitic mobility for pervasive sensor networks," in 3rd Intl Conference on Pervasive Computing (PERVASIVE 2005), Munich, Germany, May 2005.

[10] "V. Shnayder, B. rong Chen", "Sensor networks for medical care," Harvard University, Tech. Rep. Technical Report TR-08-05, April 2005.

[11]" M. Ali, T. Suleman', "MMAC: A mobility-adaptive, collision-free mac protocol for wireless sensor networks," 24th IEEE IPCCC'05, Phoenix, Arizona, USA, April 2005. 
[12] 'D. Kotz, C. Newpor', "Experimental evaluation of wireless simulation assumptions," ACM/IEEE International Symposium on Modeling, Analysis and Simulation of Wireless and Mobile Systems (MSWiM), October 2004.

[13] 'K. Langendoen, A. Baggio', "Murphy loves potatoes: Experiences from a pilot sensor network deployment in precision agriculture," in 14th International Workshop on Parallel and Distributed Real-Time Systems (WPDRTS), Rhodes, Greece, April 2006.

[14] ''S. Kurkowski, T. Camp', "MANET simulation studies: the incredibles," SIGMOBILE Mob. Comput. Commun. Rev., vol. 9, no. 4, 2005.

[15]"Mac simulator

0.2.2: http://www.consensus.tudelft.nl/software.html

[16] 'P. Levis, N. Lee', "TOSSIM: accurate and scalable simulation of entire tinyos applications," in ACM Sensys'03. New York, NY, USA: ACM Press, 2003.
[17] 'LL. Girod, T. Stathopoulos", "A system for simulation, emulation, and deployment of heterogeneous sensor networks," in ACM Sensys'04. New York, NY, USA: ACM Press, 2004.

[18] 'M. Neufeld, J. Fifield", "SoftMAC—flexible wireless research platform," in Proc. HotNets-IV, College Park, Maryland, USA, Nov. 2005.

[19] 'R. Szewczyk, J. Polastre', A. Mainwaring, and D. Culler, "Lessons from a sensor network expedition," in Proc. EWSN 2004, Berlin, Germany, Jan. 2004.

[20] 'H. Ritter, J. Schiller', "Experimental evaluation of lifetime bounds for wireless sensor networks," in Proc. EWSN 2005, Istanbul, Turkey, Jan. 2005. 